\title{
Energy growth, complexity and efficiency
}

\author{
Franco Ruzzenenti* and Riccardo Basosi*o \\ *Center for the Studies of Complex Systems, University of Siena \\ ${ }^{\circ}$ Department of Chemistry, University of Siena \\ Italy
}

\section{Introduction}

Over the last two centuries, the human capacity to harness energy or transform heat into work, has dramatically improved. Since the first steam engine appeared in Great Britain, the first order thermodynamic efficiency (the rate of useful work over the heat released by the energy source) has soared from a mere $1 \%$ to the $40 \%$ of present engines, up to the $70 \%$ of the most recent power plants. Despite this efficiency revolution, energy consumption per capita has always increased (Banks, 2007).

The economy and society have undeniably faced an expanding frontier, and both household and global energy intensities have commonly been linked to economic growth and social progress. The rising issue of energy conservation has prompted us to consider energy efficiency as more than merely a characteristic of economic growth, but also as a cause (Ayres and Warr, 2004). We thus wonder if it is possible to increase efficiency, reduce global energy consumption, and foster economic development within an energy decreasing pattern, by separating efficiency and energy growth. In other words, by reducing efficiency positive feed-backs on the system's energy level (Alcott, 2008).

In 1865, the economist Stanley Jevons was the first to point out the existence of a circular causal process linking energy efficiency, energy use, and the economic system. Jevons was convinced that efficiency was a driving force of energy growth and highlighted the risk associated with an energy conservation policy thoroughly committed to efficiency ${ }^{1}$.

Recently the Jevon's paradox has been approached in the field of Economics and termed "rebound effect". It has been the subject of articles, research, as well as a great deal of controversy over the last two decades (Schipper, 2000). Although many economists are still sceptical as to its actual relevance, most of them have agreed on the existence and importance of such an effect. Some are deeply concerned (Khazzoum, 1980, Brookes 1990,

1 "It is very commonly urged, that the failing supply of coal will be met by new modes of using it efficiently and economically. The amount of useful work got out of coal may be made to increase manifold, while the amount of coal consumed is stationary or diminishing. We have thus, it is supposed, the means of completely neutralizing the eveils of scarce and costly fuel. But the economy of coal in manufacturing is a different matter. It is a wholly confusion of ideas to suppose that the economical use of fuel is equivalent to a diminished consumption. The very contrary is the truth (Jevons, 1965)." 
Saunders, 2000, Herring, 2006) about the overall net effect and its capacity to counterbalance the gains due to efficiency. Others, however, still believe in the net benefit of energy policies focused on developing energy efficiency, although they admit the burden of having to pay a loss of savings (Shipper and Haas, 1998; Washida, 2004; Grepperud \& Rasmussen, 2003).

The most accurate and simple definition of rebound effect is: a measure of the difference between projected and actual savings due to increased efficiency (Sorrell and Dimitropoulos, 2007).

Three different kinds of rebound effects are now widely used and accepted(Greening and Greene,1997):

1. Direct effects: those directly linked to consumer behaviour in response to the more advantageous cost of the service provided. They depend on changes in the final energy use of appliances, devices or vehicles (i.e. if my car is more efficient, I drive longer).

2. Indirect effects: those related to shifts in purchasing choices of customers, either dependent on income effects or substitution effects, which have an ultimate impact on other energy services (i.e. new generation engines are economical, then I buy a bigger car or I spend the money saved for an air conditioner).

3. General equilibrium effects: changes in market demands as well as in relative costs of productive inputs that ultimately have a deep impact in the productive structure, possibly affecting the employment of energy as a productive factor (i.e. the well known substitution of capital to labour, subsequent to a rise of labour costs, is otherwise an increase of the energy intensity of the system. Labour cost may increases relative to a subsidiary process that employs more energy to run).

The above classification displays the circular feedback process's (increasing) time lag scheme, beginning with a quick response, the altered use of energy devices due to changes in energy costs, followed by a slower mechanism, changes in purchasing choices, and finally, the long term restructuring process affecting economic factors. While direct and indirect effects have found considerable attention in the literature, general equilibrium effects remain relatively unexplored due to the uneasiness of their time scale and the variety of involved variables (Binswanger, 2001) ${ }^{2}$.

\section{The economic approach to the rebound effect}

However paradoxical the rebound effect may seem, it can be explained by classic economic theory. Energy is a derived demand because it is not the actual good purchased, but a means by which a good or a service is enjoyed. Thus, technology that is able to reduce the amount of energy employed by good or service lowers the cost of that item. It is said that efficiency improvements reduce the implicit price of energy services and, according to the basic theory of market demands, the amount of goods consumed rises when prices decrease. Happy with this explanation, economic theory focused on measuring and forecasting the rebound effect. Both econometric models and neoclassical forcasting models have been

2 “Third, changes in the prices of firms' outputs and changes in the demand for inputs caused by income and substitution effects will propagate throughout the economy and result in adjustments of supply and demand in all sectors, resulting in general equilibrium effects. By taking care of the income effect, we also include the indirect rebound effect in our analysis, but we still neglect general equilibrium effects (Binswanger, 2001). 
developed that exhibit sound results, except for the third kind of effect, that unfortunately presents many features unfit for these models (Saunders, 1992; Greening and Greene, 1997; Binswanger, 2001; Sorrell, 2009).

Forecasting models are mainly based on Cobb-Douglas production functions, with three factors of production (capital, labour, energy), and which derive market demands for these factors. Since the first attempts, calculations confirmed the existence of the effect under the assumption of constant energy prices (Saunders, 1992). Econometric based research also verified the relevance of the rebound effect and further provided valid measures of the effect in a variety of economic sectors. Such measures mainly utilize the relative elasticities of demand curves. Demand curves are built on statistical regressions in prices and quantities of goods, while elasticity is a measure of the sensitivity in demand to the variation of a good's price. Although these models may be accurate, they are all single good or service designed and are consequently viable only for the detection of direct effects.

Other models based on substitution elasticities between goods or factors as well as income elasticities have addressed indirect effects (Greening and Greene, 1997). Such contributions brought the level of detection to a whole sector of an economy or to a variety of aspects related to the process of substitution highlighted in the rebound effect like the role of timesaving technologies and their impact on energy intensities (Bentzen, 2004; Binswanger, 2001). Nevertheless, very few attempts have been made to evaluate general equilibrium effects, a task which entails the recognition of the main connecting variables of an economy, spread over a long period of time. These contributions, however, fail to describe and explain major structural changes in the productive systems that cause discontinuity in the economic relations among variables. All these models are, in fact, based on a stationary framework, and therefore neglect evolutionary changes that heighten the developing pattern of an economy (Dimitropoulos, 2007).

As a result of being the first who introduced the paradox behind the development of efficiency, Jevons' work has to be considered a landmark in this matter, for he was able to trace a line that goes beyond the mere economical, or the implicit price mechanism, explanation. He thought that any technological improvement rendering the energy source more economical would stimulate the demand for energy. Furthermore Jevons had some advanced and valuable intuitions about the role of energy sources in the economic development, as well as about the dynamic between technology, energy and the economy that were too often neglected by modern economists. His contributions are summarized as follows:

1. Fuel efficiency affects market size and shape, and not just a process of substitution among factors. He noticed that both time scale and space scale of travels changed with engine technologies making new markets or new places reachable ${ }^{3}$.

2. Features of energy sources other than efficiency are relevant for economic purposes like energy intensity and time disposal (power). He argued that what made steam

3 Such structural changes are unfit for common, wide spread modeling approaches. Is noteworthy that when Jevons was developing his analysis, consumer theory was far to come and main sectors were those of steal, mining and machinery industries. Economy was chiefly engaged in building his back bone and changes at any rate were basically structural. His view of economic processes was consequentially affected by that turmoil and can be considered, to a certain extent, evolutionary. Shipper has raised the attention on structural changes, which are, according to his opinion, hardly detectable but very important in energy demand long term pattern (Shipper and Grubb, 2000). 
vessels more economical was neither fuel efficiency (wind power is more efficient) nor unit costs (wind vessels are almost costless), but instead the availability and disposal of coal as an energy source which had an incomparable positive impact on the capital return cycle.

3. A sink or a flux of free energy becomes an energy source when there is an exploiting technology and an economic need forward. He argues that from the beginning onward, a developing process of energy sources has a fundamental role as an economic driving force and not vice versa. In other words, when economic needs are compelling, technology development is significantly accelerated and as a result, feeds back to the whole economic system.

4. Prosperity is dependent on economical energy sources, and economic development is mainly shaped by energy sources and its quantity 4 . However pessimistic we may consider this statement, Jevons meant to call for an economical austerity in order to prevent society form a hard landing due to the running out of low cost coal ${ }^{5}$. He claimed that was more recommendable a stationary economy together with social progress.

What we can therefore gain from his teachings is that there is an inner tendency of an economy to render energy sources more economical and that this is the true driving force of economic development ${ }^{6}$.

Thus, for Jevons, societal development-civilization-is "the economy of power" or the constant strain on humanity of harnessing energy in a productive way, and its "history is a history of successive steps of economy (energy efficiency, n.d.r.)." The incremental process

4 "We may observe, in the first place, that almost all the arts practiced in England before the middle of the eighteenth century were of continental origin. England, until lately, was young and inferior in the arts. Secondly, we may observe that by far the grater part of arts and inventions we have of late contributed, spring from our command of coal, or at any rate depend upon its profuse consumption" (Jevons, 1965).

5 A misleading, wide spread, opinion is that Jevons skepticism was misjudged and the rising age of oil gave proof of it; but he clearly foresaw the drawbacks of such a solution: "Petroleum has, of late years, become the matter of a most extensive trade, and has been found admirably adapted for use in marine steam-engine boilers. It is undoubtedly superior to coal for many purposes, and is capable of replacing it. But then, What is Petroleum but Essence of Coal, distilled from it by terrestrial or artificial heat? Its natural supply is far more limited and uncertain than of coal, and an artificial supply can only be had by the distillation of some kind of coal at considerable cost. To extend the use of petroleum, then, is only a new way of pushing the consumption of coal. It is more likely to be an aggravation of the drain then a remedy."

6 "The steam-engine is the motive power of this country, and its history is a history of successive steps of economy. But every such improvement of the engine, when effected, does but accelerate anew the consumption of coal. Every branch of manufacture receives a fresh impulse-hand labour is still further replaced by mechanical labour, and greatly extended works can be undertaken which were not commercially possible by the use of the more costly steam-power. But no one must suppose that coal thus saved is spared -it is only saved from one use to be employed in others, and the profits gained soon lead to extended employment in many new forms. The several branches of industry are closely interdependent, and the progress of any one leads to the progress of nearly all. And if economy in the past has been the main source of our progress and growing consumption of coal, the same effect will follow from the same cause in the future." 
of energy efficiency drives more and more energy into the system, but how does it occur? Jevons, in the following passage, provides insight into such a controversial question:

Again, the quantity consumed by each individual is a composite quantity, increased either by multiplying the scale of former applications of coal, or finding new applications. We cannot, indeed, always be doubling the length of our railways, the magnitude of our ships, and bridges, and factories. In every kind of enterprise we shall no doubt meet a natural limit of convenience, or commercial practicability, as we do in the cultivation of land. I do not mean a fixed and impassible limit, but as it were an elastic limit, which we may push against a little further, but ever with increasing difficulty. But the new applications of coal are of an unlimited character (Jevons, 1965).

\section{Complexity and Efficiency}

Jevons believed that the natural tendency of economy is to expand linearly, "multiplying the scale of former applications," up to a limit and then, to overcome such limits, the system works within itself to develop "new applications". Sketched roughly, the scheme here is: growth-saturation-innovation-growth.

Jevons found an unsuspected counterpart in a famous biologist, Alfred Lotka, who was interested in the relation between energy and evolution. Indeed there are several analogies among their theories. Lotka too believed in the need for looking synoptically at the biological system in order to understand the energetics of evolution. Lotka also shares Jevons' cyclic view of processes, which, in the case of energy "transformers," he understood to be formed by an alternation growth-limit to growth- evolution- growth" According to Lotka, the reason why this process was doomed to an ever growing amount of energy flow boiled down to the cross action of selection-evolution on the one hand and the thermodynamics law on the other. In his opinion, evolution is the result of a stochastic process and a selective pressure, and moreover, "the life contest is primarily competition for available free energy." Thus, selection rewards those species adapted to thrive on a particular substrate, and the growth of such species will divert an increasing quantity of free energy into the biological system. Those species' growth will proceed until the free energy available for that transformation process is completely exploited. The dual action of case and selection will then favor new transformers more efficient in employing the free energy still available. The developmental stages of ecological succession mirror this evolutionary energetic pattern. In the first stage of ecological succession, plant pioneering species dominate, growing rapidly, but inefficiently disposing of resources. In the climax stage,

7 "But in detail the engine is infinitely complex, and the main cycle contains within its self a maze of subsidiary cycles. And, since the parts of the engine are all interrelated, it may happen that the output of the great wheel is limited, or at least hampered, by the performance of one or more of the wheels within the wheel. For it must be remembered that the output of each transformer is determined both by its mass and by its rate of revolution. Hence if the working substance, or any ingredient of the working substance of any of the subsidiary transformers, reaches its limits, a limit may at the same time be set for the performance of the great transformer as a whole. Conversely, if any one of the subsidiary transformers develops new activity, either by acquiring new resources of working substance, or by accelerating its rate of revolution, the output of the entire system may be reflexly stimulated 
however, the most efficient species in converting resources prevail (Odum, 1997). The following passage stresses this key concept:

This at least seems probable, that so long as there is abundant surplus of available energy running "to waste" over the sides of the mill wheel, so to speak, so long will a marked advantage be gained by any species that may develop talents to utilize this "lost portion of the stream". Such a species will therefore, other things equal, tend to grow in extent (numbers) and its growth will further increase the flux of energy through the system. It is to be observed that in this argument the principle of the survival of the fittest yields us information beyond that attainable by the reasoning of thermodynamics. As to the other aspect of the matter, the problem of economy in husbanding resources will not rise to its full importance until the available resources are more completely tapped than they are today. Every indication is that man will learn to utilize some of the sunlight that now goes to waste (Lotka, 1956).

Economy and biology are both evolutionary systems and both can be approached from thermodynamics. By contrast, not all analogies are suitable. Whilst less efficient transformers like bacteria persist together with more evolved vertebrates, hence biosphere makes manifest the entire evolutionary path, economy dismisses obsolete technologies (we don't see any more steam motive engines around). So, if we abandon inefficient technologies, why isn't the net effect over consumptions negative? In other words why, if we employ more efficient devices, energy use doesn't drop? History has so far proved that more efficiency results in more energy consumption. Where does this paradox come from?

Is this paradox due to the counteractive effect of population or affluence growth over efficiency or is efficiency evolution the driving factor of economic growth? We will here attempt to show how the causality chain initiate with an efficiency improvement and that growth comes after. Growth featured by those changes affecting the economic system comparable to "new applications of unlimited character" mentioned by Jevons or an "acceleration to the revolution rate of the world engine" envisioned by Lotka.

What it is being argued here is that all those changes, or among them, those affecting the structure or delivering brand new technologies into the system, may be regarded as a leap of complexity occurring to the system. Complexity, in the acceptation of organizational complexity, if it was observed as a feature of whatsoever of a system, has always displayed a high energy density rate. This means that growing complexity implies growing energy consumption. That is to say, a more complex system consumes more (more connections, more variety, more hierarchical levels). It is therefore possible that the energy saved by new and more efficient processes is absorbed or perhaps a better word, dissipated, by a more complex system. Energy savings resulting from increased efficiency would then be offset by an organization restructuring process within the system.

\section{Evolutionary Pattern}

We have advanced the hypothesis of the existence of a common, recursive pattern in evolutionary systems. This pattern underlies a broad, complex thermodynamic process involving the entire system and arises from forces embedded within the system. We have described this pattern as the following circular process: growth-saturation-complexity leapgrowth and can be depicted it as a circular process. 


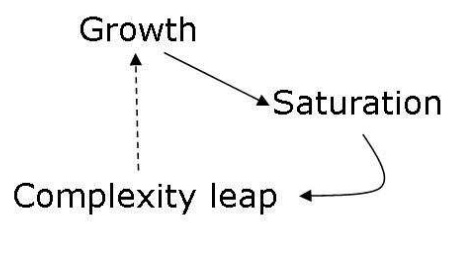

Fig. 1. Evolutionary Pattern

The growth stage relies on the presence of inner forces that drive the system to expand while seeking survival and reproduction. These forces are species (the genome) in the domain of biology, and firms (the capital) in the economy. Although it is clear how these autocatalytic processes cause the system's expansion, it is less clear how, coupled with efficiency improvements, they can divert more energy into the system or in the words of Lotka, "maximize the energy flow." It must be kept in mind that neither Lotka nor Jevons claims that the overflow of energy is the actual aim of system components. It is rather a result of their interaction with each other and with the environment. Lotka, for example, believes that two main thermodynamic strategies are adopted by organisms in order to adapt to the environment: maximizing output (power maximum) and minimizing input (efficiency maximum). The former is developed by species thriving in resource abundance and the latter by organisms struggling in scarcity conditions. According to Lotka, by pursuing unexploited free-energy more energy is driven through the system thus maximizing global output. The dichotomy between efficiency and power is therefore quite apparent ${ }^{8}$.

And there is indeed something well founded in this revelation, which is rooted in thermodynamics. The antagonism between efficiency and power is less evident from a thermodynamics perspective, meaning that if other factors are left unchanged, an efficiency improvement always leads to empowerment. The misunderstanding and thereby the paradox of efficiency comes from two major misconceptions, which can be outlined as follows:

- Thermodynamic efficiency, from the Carnot Engine onward, concerns the conversion of heat into work, not just the mere transformation of one form of energy to another.

- Efficiency, as a rate between output and input or benefits and costs, pertains to a static analysis despite the fact that the conversion process actually takes place in time and therefore costs and benefits also depend on the time elapsed.

8 There is a simplification of Lotka's vision of the energetics of evolution that states that two strategies would top evolutionary thermodynamics: one that maximizes work over time (power) in the case of resource abundance and another that minimizes energy consumed per for amount of work delivered (efficiency) in the case of scarcity. These two strategies have been summarized in the "maximum power principle," despite Lotka himself being reluctant to adopt any lofty and ambitious term like "principle" for his thinking. Moreover, in this formulation, scarcity and abundance are unrelated whatsoever to magnitude, while Lotka clearly stresses what scarcity must be compared to: the ability of a transformer to get hold of free energy and its growing rate. What are indisputably scarce or plenty are nutrients, row materials or water, which eventually affect energy efficiency. 
The first statement assumes the custom of considering conversion rates, such as the transformation of chemical energy into heat, as thermodynamic efficiencies. As previously noted, most of the controversies surrounding the rebound effect in the residential sector arise from the misleading concept of efficiency. The rate of transformation of chemical energy into heat in e.g. a bomb calorimeter is a calorie while out of the laboratory, it is a thermal efficiency, and should not be considered a thermodynamic efficiency because no work is involved 9 . The theoretical apparatus we have so far employed is therefore inapplicable. Only work needs an entropy change into the (work) reservoir in order to be dissipated while a heat sink is of unlimited disposal to the environment. In other words, the system's structure needs to change in order to dissipate (more) mechanical work, but not the same can be said for heat. This kind of efficiency, known as thermal efficiency, has much more to do with squandering. When a process becomes more thermodynamically efficient, more work is extracted from the same amount of energy (heat) and when it becomes more thermally efficient, less heat for our purpose is wasted from a heat source.

\subsection{The Time Variable Determines the Efficiency Level}

In the second statement, the attention is focused on a theoretical aspect that needs a formal treatment to be understood. It is indeed very difficult to intuitively sense that, in physics terms, a system that improves its efficiency also enhances its power. It is even more difficult to see how this can be true if a trade off exists between power maximization and efficiency optimization. A system that maximizes its efficiency actually minimizes its power and vice versa. Thus, if we improve the efficiency, we increase the power. Nevertheless, if we seek the best efficiency, we have to set the minimum power output. Is this a paradox? In a sense, yes, but only if our analysis is oblivious to the passage of time.

We have formulated two assertions in apparent contradiction. The first is that when thermodynamic efficiency improves, power increases. This direct relationship is evident by observing the definitions of efficiency and power:

$$
\eta=\frac{W}{Q_{h}}, P=\frac{W}{\Delta t}
$$

As long as the specific consumption - the rate at which the energy source is depletedremains constant, the power increases. It is noteworthy that this relationship strictly relates to the capacity of the system to draw from a particular source. The capacity depends on the specific consumption:

$$
\frac{\partial Q_{h}}{\partial t}
$$

The specific consumption is the rate of depletion of the energy source or the amount of input (fuel) per the unit of time. It reflects the capacity of the system to convey energy

9 Thermodynamic efficiency concerns the transformation of heat into work. Other non-thermodynamic efficiencies are, for example, heat transport and heat regulation or the cinematic chain. Nevertheless, any kind of efficiency can contribute to the overall thermodynamic efficiency, when a work output is obtained out of heat. 
throughout the process.The second assertion that there exists a trade-off between efficiency and power needs more mathematics to be explained. It will be illustrated by means of a Carnot Cycle, revisited with the addition of the time variable. In the Carnot Cycle, to achieve the maximum efficiency, the isothermal expansion and compression (Figure 2), need to occur at an infinitely slow speed in order to maintain an infinitesimal temperature gradient between the working substance $\left(T_{h w}, T_{c w}\right)$ and the heat reservoirs $\left(T_{h}, T_{c}\right)$. Under these circumstances, the power of the machine approaches zero since it takes infinite time to produce a finite amount of work. To speed up the process, we need to increase the gradient since the heat transfer rate is proportional to it. To thereby get more than an infinitesimal amount of power from a Carnot Engine, we have to keep the temperature of its working substance below that of the hot reservoir and above that of the cold reservoir.

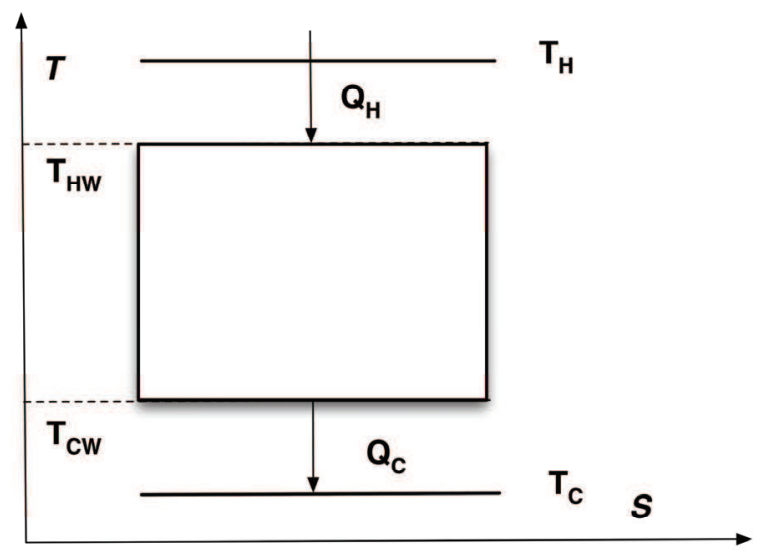

Fig. 2. Carnot Cycle

The more we increase the two gradients, the closer the extreme temperatures of the working substance. Ultimately, the two isothermal stages take place with no change in the temperature of the working substance. Heat flows directly from the hot source to the cold sink and no work is done. Hence the power output is zero and the engine has zero efficiency as well. In this model, we consider a Carnot Engine with a working substance absorbing heat from the hot source at $\mathrm{T}_{\mathrm{hw}}$ and releasing heat to the cold source at $\mathrm{T}_{\mathrm{cw}}$. Under most circumstances, the rates of heat transfer will be proportional to the temperature gradients. We assume the constant of proportionality ( $\mathrm{K}$-meaning that heat absorption/release occurs in the same conditions) and the same $\Delta t$ for the expansion and the compression ${ }^{10}$. We also assume that the two adiabatic transformations remain unaltered. We now have the following equations describing the once isothermal processes:

$$
\frac{Q_{h}}{\Delta t_{1}}=k\left(T_{h}-T_{h w}\right)
$$

10 These assumptions can be abandoned without changing the results of the model, see Curzon and Ahlborn (Curzon and Ahlborn, 1975). 


$$
\frac{Q_{c}}{\Delta t_{2}}=k\left(T_{c w}-T_{c}\right)
$$

$\mathrm{T}_{\mathrm{h}}=$ temperature of the hot source, $\mathrm{T}_{\mathrm{c}}=$ temperature of the cold source, $\mathrm{T}_{\mathrm{hw}}=$ max temperature of the working fluid, $\mathrm{T}_{\mathrm{cw}}=$ min temperature of the working fluid

Since the remaining two processes are adiabatic, they follow the relation (5):

$$
\frac{Q_{h}}{T_{h w}}=\frac{Q_{c}}{T_{c w}}
$$

The power of the system will be defined in equation (6):

$$
\begin{gathered}
P=\frac{W}{2 \Delta \mathrm{t}} \\
W=Q_{h}-Q_{c}, \Delta t_{1}=\Delta t_{2}
\end{gathered}
$$

The maximization of the power, as a function of $\mathrm{T}_{\mathrm{hw}}$, the hotter working temperature, will give the following result for the optimum power output:

$$
T^{h w}=\frac{1}{2}\left(T_{h}+\sqrt{T_{h} T_{c w}}\right)
$$

at a corresponding efficiency of

$$
\eta=1-\sqrt{\frac{T_{c}}{T_{h}}}
$$

It will be useful to do a variables' substitution to depict the trade off so we now fix $\mathrm{x}=\mathrm{T}_{\mathrm{cw}} / \mathrm{T}_{\mathrm{hw}}$. According to this model, the efficiency-power trade off can be sketched as function of $x$ and whereby Carnot efficiency will be represented by curve (10) and power output curve (11):

$$
\begin{gathered}
\eta=1-x \\
P=\frac{k}{4}\left(T_{c}+T_{h}-T_{h} x-\frac{T_{c}}{x}\right)
\end{gathered}
$$

The two curves can be plot in a graph, assuming $\mathrm{T}_{\mathrm{h}}$ and $\mathrm{T}_{\mathrm{c}}$ of 300 and 25 degree Celsius; and fixing $\mathrm{k}$ at 0.05 (Fig.3). To reach the maximum theoretical efficiency ( $\eta$ for the isothermal transformation) the system must approach thermal equilibrium and therefore maximum 
slowness. Since it arises from power maximization, the optimal output will be somewhere between theoretical maximum efficiency and zero efficiency and it will only be determined by the sources' temperatures $\left(T_{h}\right.$ and $\left.T_{c}\right)$. So for every boundary condition in a Carnot Cycle, there is a single optimal value of output. Even if we abandon most of the abstract assumptions about the Carnot Cycle thus introducing further irreversibility, the peak of the curve will probably shift, but the trade off is unavoidable. We have to set the engine at either maximum efficiency or maximum power. "However, when the cost of building an engine is much greater than the cost of fuel (as is often the case), it is desirable to optimize the engine for maximum power output, not maximum efficiency (Schroeder, 2000)."

$$
\frac{k}{4}\left(T_{c}+T_{h}-T_{h} * \mathrm{X}-\frac{T_{c}}{x}\right), \mathrm{k}=0.05, T_{c}=293, T_{h}=593
$$

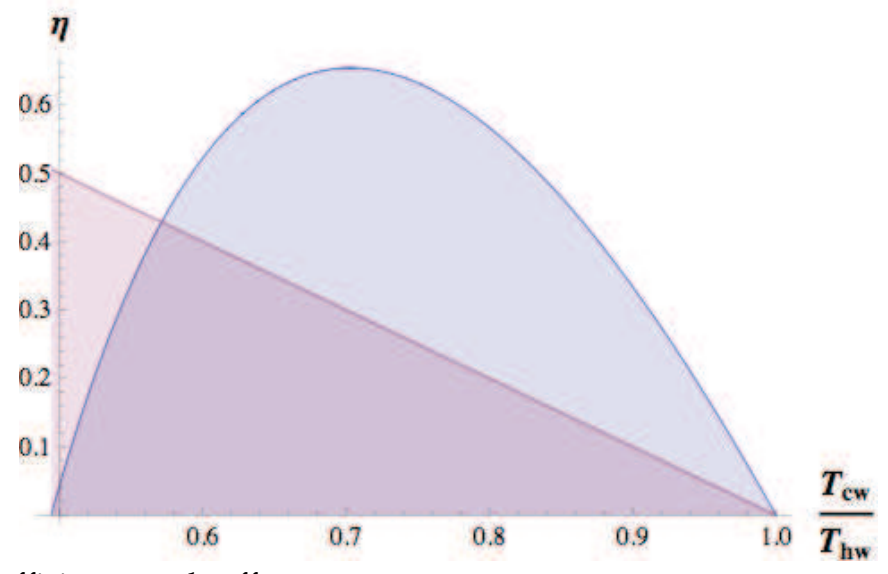

Fig. 3. Power-efficiency trade off

The power maximization will lead to sub-optimal efficiency (with respect to Carnot efficiency) which depends on sources' temperatures with the explicit relation (9) while Carnot efficiency is:

$$
\eta_{\text {Carnot }}=1-\frac{T_{c}}{T_{h}}
$$

It is noteworthy that such an efficiency level seems to be much closer to the running efficiency of most of energy converting sources than the Carnot efficiency (Table 1).

\subsection{Efficiency improvement and power enhancement}

We can further assume that efficiency improvements also apply to engine parts, in addition to working temperatures ${ }^{11}$. Any technical improvement concerning the material employed

\footnotetext{
11 If we consider sources' temperature changes, we return to the dominion of Carnot efficiency while if we take into account working temperatures, we resort to the efficiency-power trade off sketched by the model.
} 
or the reduction of friction would lead to a higher $\mathrm{K}$ and a better (faster) heat transfer across the machinery12. Since $\mathrm{K}$ does not affect the output regulation (the maximum value is not dependent on $\mathrm{K}$ ), this will in turn, increase the rate of $\mathrm{Q}_{\mathrm{h}}$ and the power. According to the value of the maximum power, it is clear that any increase in $K$ (given $T_{h}$ and $T_{c}$ ) will augment the power by a factor of $1 / 4$, shifting the peak upward. More efficiency will therefore lead to higher power.

Suppose we want to increase efficiency as much as possible, leading the control parameter $\mathrm{K}$. We may push further $\mathrm{K}$, in order to increase the heat transfer rate and get an higher efficiency, but we will end up moving away from the theoretical maximum efficiency level, toward an higher power output, as it is shown in the animation ${ }^{13}$.

Through this model, we have shown how the efficiency-power paradox is apparent and we have also described the thermodynamic conditions of the efficiency-power trade off. We can thus draw Lotka's conceptual framework of power maximization versus efficiency optimization in the context of the economy of power. It is, as we have already highlighted, an economic optimization that leads to maximum output ${ }^{14}$. Whenever the cost of fuel is relatively less constraining than the cost of machinery, power will be maximized. Nevertheless, every efficiency improvement involving technological development will probably lead to a more complex engine (or process) and therefore, will, on the one hand, reduce the relative price of energy, but on the other, raise the cost of the apparatus. This will ultimately amplify the bifurcation and positively feed back to the optimum power level15.

\footnotetext{
12 The paradoxical effect of increasing both efficiency and power can be easily understood if we think energy as space integral and work as the time integral of force. A process that reduces energy input in less time, increases power, as integrations over the same function are not independent. That is to say: if we use less energy per unit of space, and unit of time, in the same amount space we will save energy, yet in the same time lag we may use more energy!

${ }^{13}$ Animation at: http// sciyo.com

14 Concepts of the like of "costs" and "economic optimization" should not be intended in a strict way. Broadly speaking, costs are to be meant as thermodynamic cost.

15 The idea of sub-optimal efficiency level output was investigated in the filed of biological systems. As early as the 1955, Hodum and Pinkerton (Hodum H.T., Pinkerton R., 1955) published an article in which, adopting Lotka approach an vision for life's energetics, tried to demonstrate that "natural systems tend to operate at that efficiency which produces a maximum power output". Such efficiency was lower then the maximum attainable and, according to them, was exactly of 50\%. "In natural systems there is a general tendency to sacrifice efficiency for power output". The idea of the $50 \%$ set point was based on the finding that most of energy converting systems were featured by coupled antagonists processes. "The essence of biochemical workings of an organism is the coupling of an exergonic catabolism to an endergonic anabolism that results in growth, reproduction and maintenance". Although this paradigm may account partially or even totally, for the derivation of the $50 \%$ value of efficiency, it was a striking intuition. It is remarkable, for example, that in the former model, whereas it is not so evident, there are two counteractive processes: the heat absorption an then heat release. The heat disposal affect the power output as much as the heat intake, as we know empirically, from electric power plants. Thus, this simple thermodynamic model resembles, by this point of view, the "living systems" of Odum theory. Conversely, as already Odum didn't fail to mention later on (Odum, 1983), the article of Cuzon and Ahlborn of 1975, on which this model is based, gave a sound evidence and a formal basis, to the postulate of the "maximum power principle"
} 


\begin{tabular}{|l|l|l|l|l|l|}
\hline Power Source & Tc & Th & $\mathbf{\eta}$ (Carnot) & $\mathbf{\eta}^{*}$ (model) & $\mathbf{\eta}$ (observed) \\
\hline Coal Fired Steam Plant & 25 & 565 & $64.10 \%$ & $40 \%$ & $36.00 \%$ \\
\hline Nuclear Reactor & 25 & 300 & $48.00 \%$ & $28 \%$ & $30 \%$ \\
\hline Geothermal Steam Plant & 80 & 250 & $32.30 \%$ & $17,5 \%$ & $16 \%$ \\
\hline
\end{tabular}

Table 1 Source: Cuzon and Ahlborn, 1975.

\subsection{The Case of Trucks}

The truck industry and therefore, the road freight transport sector, gives a useful example of empowerment brought about by the efficiency improvement of the engine and vehicle technologies. From the late 1970's onward, efficiency rose steadily as an effect of technology research that tried to overcome the effects of soaring energy costs. Initially, such improvements were employed to reduce consumptions, but later technology development partially addressed power enhancement. Energy efficiency, as measured by fuel economy distance travelled, at constant speed, for unit of fuel consumed, increased since late 1970's to late 1990's of about 30\%. However, if we rescale fuel economy to the power shift of engines (adjusted fuel economy), we can observe a major change in efficiency (Ruzzenenti and Basosi, 2009a). This is also evident from the comparison of two trends of fuel economy and adjusted fuel economy (fuel economy divided by the engines' power) for a sample of 97 different European heavy-duty trucks. Initially the two metrics are coupled and show how efficiency was employed to reduce fuel consumption; we can see a dramatic drop in both fuel economy and adjusted fuel economy. Later trends display a sharp bifurcation, from mid 1990's onward, that explains how efficiency was then employed to enhance power and reduce consumptions (Figure 3 )

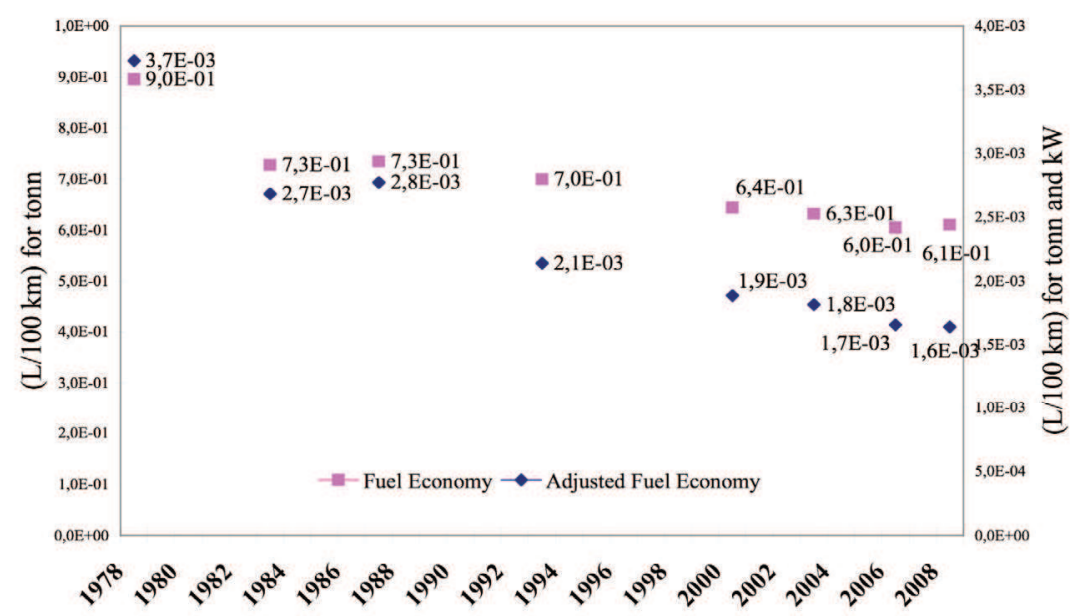

Fig. 4. Efficiency and power bifurcation in European Truck Industry (Ruzzenenti and Basosi, 2009a) 


\section{Structural Complexity}

The underlying hypothesis of this work is that higher complexity counterbalances, on a global scale, the effects of higher efficiency on a process scale. It is our general understanding of evolution that selection operates by reward complexity. More complex, in the context of biology is often used as a synonym for fittest in terms of the competition for resources. Technological advances also develop from less to more complex devices. The meaning of complexity has never been questioned, for it has been evident in the semantic of nature or progress since earliest observations. A eukaryotic cell is more complex than a prokaryotic one and a Ferrari F1 is more complex than a Ford T. Under this perspective, complexity is countable, if not measurable, by the number of different components, parts or organs. If we abandon the conviction that progress always evolves toward higher complexity, that is to say, if we relinquish the belief of an immanent trend in nature; or if we are dealing with systems that differ in structure rather than in number of components, how can we apply such well established knowledge?

It is beyond the goals of this analysis to establish what complexity is or how it should be approached. The scientific community has been unable to establish or agree on a universal definition or paradigm of complexity and any attempt to univocally measure complexity is therefore doomed to failure ${ }^{16}$. It will be here assumed that a more complex system has a higher energy density or in other words, consumes more energy per unit of mass and time. There is a great deal of evidence, from biological records to cosmological entities, of such a relationship and therefore we believe it can be considered a reasonable assumption.

Indeed, this strongly recursive pattern in nature -linking energy density and compolexity, caused many scientists to think that energy could itself be considered a measure of complexity (Odum H.T., 1996; Odum E.P., 1997; Chaisson, 2001). Let's assume a more complex system consumes more (per unit of mass and time) and the complexity we refer to, it is a structural or morphological complexity, as we are dealing with systems with undefined boundaries and innumerable components, like the productive and transport systems. The two main assumptions regarding complexity that we are concerned with are:

1. A more complex system consumes more energy per unit of mass and unit of time (higher energy density rate)

2. Structural complexity primarily concerns the components' organization $^{17}$ rather than the components' variety or number

16 "By 'complexity', we refer to the term intuitively as used in ordinary discourse, a definition culled from many sources: 'a state of intricacy, complication, variety, or involvement, as in the interconnected parts of a structure-a quality of having many interacting, different components.'In this work we shall come to identify complexity in two operational ways: as a measure of the information needed to describe a system's structure and function, or as a measure of the rate of energy flowing through a system of given mass. No attempt is made here to be rigorous with the words 'order', 'organization', 'complexity', and the like; this is not a work of classical philology or linguistic gymnastics. Indeed, no two researchers seem able to agree on a precise, technical definition of such a specious word as complexity, which may be context-dependent in any case (Chaisson, 2001)".

${ }^{17}$ For organization we refer to any system's components acting or arranged in a cooperative, systematic fashion. 
Further remarks attain the duality efficiency/complexity. We should bear in mind that while we are referring to energy efficiency improvements, we are dealing with a processscale analysis, whilst the leap in complexity concerns the global-scale analysis. These phenomena are at two different hierarchical levels:

1. Energy efficiency concerns energy converting processes and is therefore at the components level of the system

2. Complexity (structural) concerns the organization of the system and is thus at the global level of the system

We try to hereafter relate energy efficiency enhancement to complexity change. To accomplish this, we have to detect changes in system organization that move in the direction of higher complexity. Yet, changes in which system?

Since we have been dealing in the case study with truck efficiency, it would make sense to refer to the freight road transport system, but that would be misleading because the goods transport sector is merely a sub-component of the whole productive system. Transport service is just a derivative demand, which means, in economics terminology, that someone wants a good to be moved from one place to another. The shipment is the means, not the goal. Our analysis therefore has to address the productive system in order to detect long term changes in transport demand. Transport demand is derived from the needs of the productive system. The transport system and the productive system, under the scope of present analysis, are two parts of a whole.

\subsection{Complexity leap: structural analysis}

The main feature of the shift from a fordian to a post-fordian system concerns the location of the productive chain. Formerly, the productive chain was set entirely in one site, to which raw materials were delivered and from which products were shipped. From the 1970's onward, big firms began disassembling the production chain and redistributing it over several scattered structures, belonging to the same company, or, more generally, belonging to other international firms or local producers system. As a matter of fact, the productive chain changed shape thereafter and it changed in such a fashion that the complexity of the structure increased. It can be shown, by means of graph theory, that the post-fordian structure increased in connectivity and path-cycles diversity across its nodes (Ruzzenenti and Basosi, 2008b). Hence the post-fordian structure presents a higher degree of freedom and thus relates to a more complex system. A system with a higher degree of freedom is a more complex system in the sense that, as for any physical system, it has increased multiplicity or number of different available states. In other words, a more complex system has more ways to arrange the components, in this case goods or raw materials, and therefore to dissipate energy.

According to the hypothesis here advocated, complexity increases when the system can rearrange its components in such a manner that the number and the path length, or the speed of interactions, will be augmented within the same boundaries ${ }^{18}$. That is to say, complexity growth consists of an intensive rather than an extensive change, affecting the

18 It noteworthy that, in a network, the number and the path length must be considered intensive features as they can grow without affecting the extension of the network, which is determined eventually only by the number of nodes (components). 
internal structure of the system, which may be expressed by a new arrangement of system components ${ }^{19}$. This hypothesis expresses view of complexity based on the concept of geometry 20 .

In our opinion the network structure development, that eventually results in complexity growth at any system level, is the outcome of forces (energy influx driven by autocatalytic processes) in the context of hindering boundary conditions. It is the simple growth (in extent and in number of components) the normal behavior. That is to say, without hindering boundary conditions, the system expands its structure, qualitatively unaltered (spatial growth). The system develops in a primary and spatial manner initially, then, when saturation is reached, in a secondary and geometrical (structural) one. It is such geometrical development that enables the system to increase its degree of freedom and to host more energy (or energy density rate) within the same constraints. When this complexity change emerges, the incoming structure, albeit already available to system components, becomes now more probable. The boundary conditions ultimately determine the likelihood of the new structure. It is therefore the role played by saturation in system's growth that must be addressed in order to understand the surge of complexity leap.

After the first oil crisis, worldwide industrial production dramatically decreased. There are many clues, indeed, that industrial production at that time reached a saturation point. Statistics show that between the early 1970's and 1990's, a revolution occurred in economic and societal structure that might be considered the end of the industrial age (IEA, 1997). Until that time, linear growth lasted for about 20 years and consisted of a shift in the active population (which was itself growing) from agriculture to industry. It was the nature of the "economic boom", the linear "growth in extent and numbers" (Lotka, 1956). The birth rate thereafter inverted its trend (also in relation to the average income) and population employed in industry reached a maximum and started decreasing (Ruzzenenti and Basosi, 2009b). Industry received a dramatic set back and consequently began to explore new strategies to reduce labour costs and regenerate production. The structural change we have been describing thus far-the globalization and outsourcing revolution-took between 10 and 15 years to become established and influential. However, after the 1990's, the growth trend in the industrial sector resumed and the economy retrieved.

\subsection{Degree of freedom reduction/increase}

When analyzing the structural complexity change resulting from globalization, of paramount importance is the shift from a uni-located, national productive chain to one that is pluri-located and international. For those firms relying on external resources to pursue their productive needs, production became less costly, but more subdued due to uncontrollable factors. Part of its activity, formerly controlled managerially and internally,

19 An example in cells is represented by the internal skeleton of microtubules that increase the speed of molecules across the cell compared to a transportation system based on simple diffusion. In ecosystems, furthermore, there are food chains and predator-prey dynamics that represent another "transportation network" over which matter flows faster.

20 According to Lotka, geometry is a prominent feature of thermodynamics of living systems and thus, of a sort completely different from those normally addressed by equilibrium thermodynamics. Whereas the latter mainly deals with "structure-less systems", of the like of chemical coefficients, the former must deals with the "geometrical features" of the system (Lotka, 1956). 
was then focused on the free market. This shift reduced the stability of the firm and reduced its degree of freedom (choices of allocation). After globalization, firms could explore labour costs according to various national legislations and average incomes. The same occurred for financial and fiscal conditions or the proximity to productive districts. The system (entire market) could thereby reduce production costs by selecting where to set plants or rely on suppliers. It is in this sense that globalization produced the rise of new spatial gradients in the productive system. The whole system thereby increased its degree of freedom. We face therefore the counteractive interplay of degree of freedom, on two hierarchical levels, triggered by a saturation stage. To better stress how this interplay of degree of freedom, working in opposite directions, can be caused by saturation, it is best to approach physical systems for analogies.

For example, if we increase the pressure of a gas in a specific volume, we reduce its degree of freedom and it consequently can become a liquid, at certain temperatures. At the same time, when a liquid changes its motion regime, as in Benárd cells, from a pure, random dissipative system (Figure 5A) to a global dissipative one (Figure 5B), which displays features several magnitudes larger than molecules, a superstructure can arise that was previously available, but very unlikely.

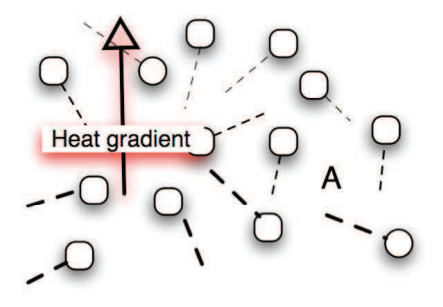

Random motion of gas molecules

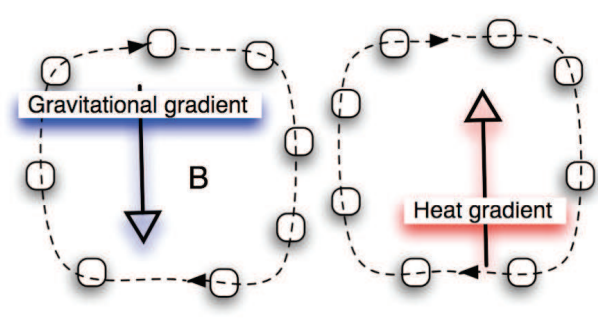

Convection cells in a liquid layer

Fig. 5. Degree reduction/increase in dissipative structures (Ruzzenenti and Basosi, 2009b)

Gravity and viscosity constraints make such a structure, beyond a certain level of energy input, possible. The Benárd cells phenomenon is indeed possible when the gradient temperature and water level thickness are known, but not when the vessel permits the fluid to dissipate heat in random motions. In other words, the boundary conditions together with the pressure imposed upon the system by an increasing energy flux, changes the macrostate (energy density) of the system by modifying its microstates (the molecular motion). The random motion of molecules reflects one gradient, the temperature, which is not spatial (geometrical), while the superstructure is exposed to the spatial gradient. That is to say, while the first gradient is defined by one variable, the latter is described by three variables and probabilities consequently change. Dissipation into one variable is therefore more probable than onto three variables, unless boundary conditions render the former impossible. In Benárd cells, such conditions are exemplified in Van der Waals forces, the low heat capacity of water, and restrained vessel thickness (Chandrasekhar, 1961; Prigogine and Stengers, 1984; Swenson, 1997). The connectivity recasts the same trade off in a network system's conceptual framework. A network system grows in complication as long as a new 
component is connected on the same hierarchical level and it grows in complexity when a new component is introduced on a higher hierarchy (Allen and Starr, 1982). The emergence of a new hierarchy involves coherent behavior for lower level components to the same extent as molecules in Benárd cells, and most importantly, the onset of a new spatial gradient for the higher component, which must now recognize system boundaries. On a molecular scale, cells in the body behave like a network. From the stand point of the organism, however, they act as a whole unit. Indeed "free" cells in substrates are mainly exposed to chemical gradients (temperature, pressure and gravitational gradients as well), while "embedded" cells in tissues that form organs are described by spatial, three dimensional, gradients.

\subsection{Spatial symmetry rupture}

We believe economic systems (and macroscopic complex systems in general) can exhibit a similar evolutionary pattern: a space symmetry rupture emerges from compelling boundary conditions and increasing energy inflow. In the case of the productive structure's evolution it can be shown that space was isotropic ${ }^{21}$ in the former state (fordian) and non isotropic in the latter (post-fordian): a spatial symmetry breaking occurred (Figure 6). What made this spatial gradient rise was a reduction in firms' degree of freedom production settings, coupled with an energy efficiency leap. More energy was thus available to the system amid a condition of hindering forces applied to its boundaries. Two counteractive forces are beneath a symmetry rupture. In this case the symmetry rupture put a space gradient upon the system, with which it induces its variables (components) to organize themselves. Globalization and outsourcing set production plants in a new, oriented space that was formerly homogeneous.

We would like now to clarify the reason why it has been used the word rupture has been used in place of breaking to describe the symmetry change. The concept of "symmetry breaking" applies to the temporal scale, whereas here space symmetry has been considered. That is to say, the time-symmetry concept concerns a sudden change in the developmental path of the system; nevertheless this change affects the system itself, rather than the space of the system. In Prigogine's paradigm a dynamic system is considered and it is thus described by a dynamic function, whereas, in the symmetry rupture a phase transition rather than a dynamical, however non-linear, change is described ${ }^{22}$.

\footnotetext{
${ }^{21}$ In other words, there is just one way to go from the periphery to the centre, regardless of the number of nodes considered, while there are many ways to connect the same number of points in the path. Furthermore, the number of different ways increases with the number of points. This does not mean that, in a scattered productive chain, factories (points), are connected randomly, but instead means that there are multiple ways for a chain to develop its pattern and just one for a centralized system.

22 "We see therefore, that the appearance of a periodic reaction is a time-symmetry breaking process exactly as ferromagnetism is a space-symmetry breaking one. [....] To understand at least qualitatively this result let us consider the analogy with phase transitions. When we cool down a paramagnetic substance, we come to the so-called Curie point below which the system behaves like a Ferro magnet. Above the Curie point, all directions play the same role. Below, there is a privileged direction corresponding to the direction of magnetization "(Prigogine, 1977).
} 


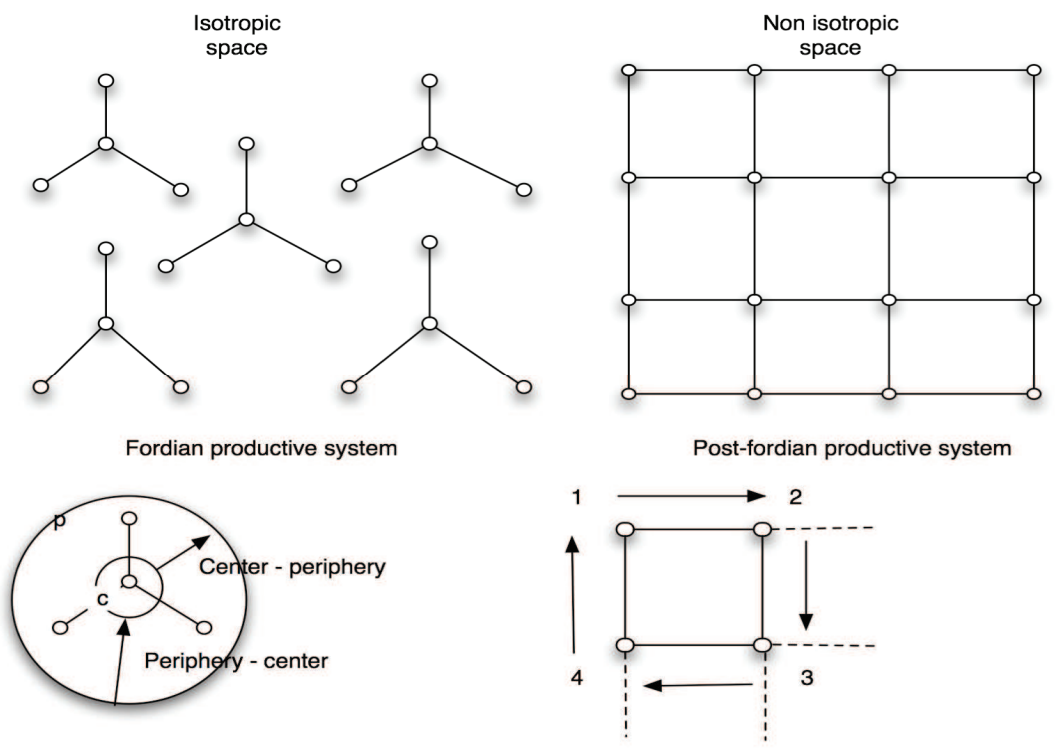

Fig. 6. Spatial symmetry and productive chain (Ruzzenenti and Basosi, 2009b)

It is noteworthy that Prigogine used the concept of space-symmetry breaking as a metaphor to introduce the new concept of time-symmetry breaking. Now, we want to retrieve the concept of space symmetry breaking (symmetry rupture in the jargon so far adopted) as we think it is fundamental to understand how evolution may concern the space of the system, rather than the system itself. Furthermore, it should also be noticed that the concept of space-symmetry breaking includes the concept of time-symmetry breaking and not vice versa.

\subsection{Time scale and Spatial scale}

Complex systems display a spatial gradient which is sometimes many orders of magnitude larger than gradients involving the scale of components. This important feature of complexity was first envisaged by Prigogine. Parameters describing dissipative structures, like Bénard cells, are macroscopic compared to parameters describing structures at thermodynamic equilibrium. Indeed, while crystals are described by interactions of the order of 10-10 meter, convective cells display a size of the order of the 10-2 meter (Prigogine and Stengers, 1984). The same can be said for the characteristic time of phenomena. Time scale varies greatly for the above mentioned systems: the vibration period of molecules is of the order of $10^{-15}$ seconds whereas convective motions have a period in the order of seconds. It is noteworthy that this scale effect, consequential to the hierarchical leap, seems to be a common feature of all complex systems, spanning from simple, non-living dissipative structures to greatly complex human-made and biological systems. In the case study here presented the magnification of time scale is clearly established in the nature of decision process that characterize the view-point of firms. As long as the entire chain was engulfed in the same firm, if not in the same production plant, any decision inherent to the volume of 
the production was almost readily attainable. Outsourcing, conversely, brought the productive chain outside the firm, making the setting of the plant an endogenous variable and the volume of the production an exogenous variable (or at least a less controllable one). Nevertheless, decisions having to do with production's settlement develop themselves over a much larger range of time.

Obviously, the time lag scales up due to the spatial extent of interactions, which increases many orders of magnitude throughout hierarchy as components' size remains the same. This is why the time scale is commensurate to the spatial extent of the system. Nevertheless it seems that magnification of the time scale is affected by space in a fashion that is not entirely reducible to an extensive factor. Time scale as it grows displays a cyclic phenomenon which seems to relate to the symmetry property of the space. In mechanics a cyclic system displays properties of invariance that are proportional to the symmetry properties of the space: to every local symmetry there corresponds a conservation law. A conservation law states that some quantity describing a system remains constant throughout its motion; expressed mathematically, the rate of change of its derivative with respect to time is zero.

A system that is cyclic exhibits symmetry as if the space was homogeneous. Therefore by means of cyclic patterns, symmetry in space is re-established and growth can develop again in a continuous way. It is needless to emphasize that cycles are a prominent feature of complex systems, regardless of the nature or the scale that is involved.

\section{Conclusions}

In the first part of the chapter the rebound effect -the growing energy use coupled with an efficiency enhancement, was employed to introduce the broader question that relates energy efficiency to energy density rate. It was shown that the paradox partially derives from misconception of energy efficiency and power. It must be firstly conceptually set apart thermodynamic efficiency from other forms of efficiency. It must than bore in mind that thermodynamic efficiency is strictly connected to power, in two ways. On the one hand there is power-efficiency trade-off and evolutionary systems tend to maximize power rather than efficiency. On the other hand, an efficiency enhancement normally leads to a power shift, as a side effect. In the second part of the chapter, we approached the question of the interdependence between efficiency, complexity and energy density, to illustrate how the causality chain can be reversed: efficiency leads to an higher energy density rate and eventually, to a complexity leap. A complexity leap that is underlined by a change in the space of the system.

As in a phase transition, space symmetry rupture seems to be an important aspect of complexity change. Symmetry rupture, introducing a new gradient in the system space, allows variables (components) to organize themselves. This new arrangement, on the scale of variables, reduces their degree of freedom, on the scale of the whole system, increases it. The change deeply concerns the structure, therefore the geometry of the system. Between the new and the old structure a topological change occurs. In our opinion, the topology of the system has to be addressed with graph theory. Yet, the transition between the two phases is still an open question and more research is needed. A formal analysis of it should start from recent acquisitions in the field of network theory (Ruzzenenti, Garlaschelli and Basosi, 2010). However, as we tried to illustrate in this chapter, the new arising structure will be more 
complex and more energy intensive. Higher energy density rate will be an outcome of a transition that will maximize links and frequency of interactions. Such a transition lays behind, in our opinion, the so called "rebound effect" (Jevons paradox) and explains why energy efficiency has always led to energy growth. Energy conservation policies should therefore contemplate, together with strategies prompted at fostering energy efficiency, measures directed at balancing the long term positive effect over energy consumptions due to a structural changes in economy.

\section{References}

Alcott B., 2008. The sufficiency strategy: Would rich-world frugality lower environmental impact? Ecological Economics, $64,770-786$

Allen T. H. F., and T. B. Starr. 1982. Hierarchy: perspectives for ecological complexity. University of Chicago Press, Chicago, IL.

Ayres R. U., Warr B., 2005. Accounting for growth: the role of physical work. Structural Change and Economic Dynamics ,16, 181-209

Banks F. E., 2007. The political Economy of World Energy: An Introductory Textbook. Singapore and New York, World Scientific.

Bentzen J., 2004. Estimating the rebound effect in US manufacturing energy consumption, Energy Economics, 26, 123-134.

Binswanger M., 2001. Technological progress and sustainable development: what about the rebound effect? Ecological Economics 36 (2001) 119 - 132

Brookes, Leonard, 1990. The greenhouse effect: the fallacies in the energy efficiency solution. Energy Policy 18 (2), 199-201.

Chaisson E., 2001. Cosmic Evolution-The Rise of Complexity in Nature, Harvard University Press, Cambridge, Massachusetts, London, U.K., 2001.

Chandrasekhar, S. 1961. Hydrodynamic and hydromagnetic stability. Oxford, Clarendon.

Curzon F., Ahlborn B., 1975. Efficiency of a Carnot Engine at Maximum Power Output. American Journal of Physics 41, 22-24 (1975).

Dimitropoulos J., 2007. Energy productivity improvements and the rebound effect: An overview of the state of knowledge. Energy Policy 35, 6354-6363.

Geening, Lorna A., Greene, David L., Difiglio, Carmen, 2000. Energy efficiency and consumption - the rebound effect-a survey. Energy Policy 28 (6/7), 389 - 401.

Grepperud S., Rasmussen I. 2003. General equilibrium assessment of rebound effects, Energy Economics, 2003. 189-203.

Herring H., Energy efficiency - a critical view. Energy 31 (2006) 10-20.

IEA, Indicators of energy use and efficiency, 1997.

IEA Energy Statistics Statistics on the Web: http://www.iea.org/statist/index.htm

Jevons, W. Stanley, The coal question - An Inquiry Concerning the Progress of the Nation, and the Probable Exhaustion of our Coal-mines, M.A., 1l.D., F.R.S., Augustus M. Kelley Publisher, New York, 1965

Khazzoom, J.D., 1980, "Economic Implications of Mandated Efficiency in Standards for Houshold Appliances." The Energy Journal, Vol.1, No.4, pp21-40.

Kummel, R., 1989. Energy as a factor of production and entropy as a pollution, Ecological Economics, 1 (1989) 161-180. 
Lotka A., 1956. Elements of Mathematical Biology, Dover Publications, Inc, New York. (first publication: elements of physical biology, The Williams and Wilkins Co., Inc, 1924).

Odum E.P., 1997. Ecology: A Bridge Between Science and Society, Sinauer Associates, Inc., Publishers. Sunderland, Massachusetts 01375 U.S.A.

Odum H.T., 1955. The speed regulation: the optimum efficiency for maximum power output in physical and biological systems. American Scientist. 43 (1955), 331-343.

Odum H.T., 1983. Maximum Power and Efficiency: a rebutal, Ecological Modelling, 20, 7182.

Odum H.T., 1996. Environmental Accounting. Emergy and Environmental Decision Making. John Wiley \& Sons, Inc., New York, USA.

Prigogine I., G. Nicolis, A. Babloyantz, 1972, Thermodynamics of evolution. Physics Today 25 (11).

Prigogine Y., 1977. Time, Structure and Fluctuations, Nobel Lecture.

Ruzzenenti, F., Basosi, R., 2008a The role of the power/efficiency misconception in the rebound effect's size debate: Does efficiency actually lead to a power enhancement? Energy Policy 36-9, September 2008, 3626-3632.

Ruzzenenti F., Basosi R., 2008b The rebound effect: An evolutionary perspective. Ecological Economics, 67 (2008) $526-537$.

Ruzzenenti F., Basosi R., 2009b Complexity change and space symmetry rupture, Ecological Modelling, 220(2009)1880-1885.

Ruzzenenti F., Basosi R., 2009a Evaluation of the energy efficiency evolution in the European road freight transport sector. Energy Policy, 37 (2009) 4079-4085.

Ruzzenenti, F., Garlaschelli D., Basosi R., Complex Networks and Symmetry: a Review with Applications to the Evolution of World Trade. Article in Press, pre-print: arXiv:1006.3923v1 [q-fin.GN]

Saunders, Harry D., 2000. A view from the macro side: rebound, backfire and KhazzoomBrookes. Energy Policy 28 (6/7), 439-449.

Schroeder D., 2000. Thermal Physics, an introduction to. Addison Wesley Longman.

Schipper L., Haas R., 1998. Residential energy demand in OECD-countries and the role of irreversible efficiency improvements. Energy Economics 20 (1998) 421-442.

Schipper L., Grubb M., 2000 On the rebound? Feedback between energy intensities and energy uses in IEA countries. Energy Policy 28, 367$\} 388$

Sorrell, S., Dimitropoulos, J., 2007. UKERC Review of Evidence for the Rebound Effect: Technical Report 5-Energy Productivity and Economic Growth Studies. UK Energy Research Centre, London.

Sorrell S., 2009. Jevons' Paradox revisited: The evidence for backfire from improved energy efficiency. Energy Policy, 37 (2009) 1456-1469.

Swenson R., 1997. Autokatakinetics, evolution, and the law of maximum entropy production: a principled foundation toward the study of human ecology, in: Freese, L., Advances in human Ecology, vol.\&. JAI Press, Greewich, CT, pp. 1-47; 1989. Emergent attractors and the law of maximum entropy production: foundations to a theory of general evolution. Syst. Res. 6, 187-197.

Washida T., 2004. Economy-wide Model of Rebound Effect for Environmental Efficiency, proceedings of International Workshop on Sustainable Consumption, University of Leeds, March 5-6, 2004. 


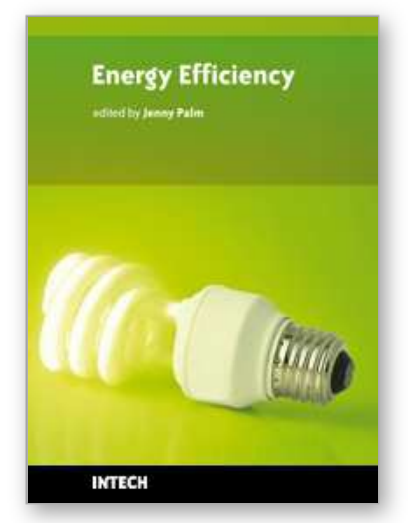

\author{
Energy Efficiency \\ Edited by Jenny Palm
}

ISBN 978-953-307-137-4

Hard cover, 180 pages

Publisher Sciyo

Published online 17, August, 2010

Published in print edition August, 2010

Global warming resulting from the use of fossil fuels is threatening the environment and energy efficiency is one of the most important ways to reduce this threat. Industry, transport and buildings are all high energyusing sectors in the world and even in the most technologically optimistic perspectives energy use is projected to increase in the next 50 years. How and when energy is used determines society's ability to create long-term sustainable energy systems. This is why this book, focusing on energy efficiency in these sectors and from different perspectives, is sharp and also important for keeping a well-founded discussion on the subject.

\title{
How to reference
}

In order to correctly reference this scholarly work, feel free to copy and paste the following:

Riccardo Basosi and Franco Ruzzenenti (2010). Energy Growth, Complexity and Efficiency, Energy Efficiency, Jenny Palm (Ed.), ISBN: 978-953-307-137-4, InTech, Available from:

http://www.intechopen.com/books/energy-efficiency/energy-growth-complexity-and-efficiency

\section{INTECH}

open science | open minds

\section{InTech Europe}

University Campus STeP Ri

Slavka Krautzeka 83/A

51000 Rijeka, Croatia

Phone: +385 (51) 770447

Fax: +385 (51) 686166

www.intechopen.com

\section{InTech China}

Unit 405, Office Block, Hotel Equatorial Shanghai

No.65, Yan An Road (West), Shanghai, 200040, China

中国上海市延安西路65号上海国际贵都大饭店办公楼405单元

Phone: +86-21-62489820

Fax: +86-21-62489821 
(C) 2010 The Author(s). Licensee IntechOpen. This chapter is distributed under the terms of the Creative Commons Attribution-NonCommercialShareAlike-3.0 License, which permits use, distribution and reproduction for non-commercial purposes, provided the original is properly cited and derivative works building on this content are distributed under the same license. 